\title{
A!
}

This is an electronic reprint of the original article.

This reprint may differ from the original in pagination and typographic detail.

Pekola, Jukka; Giazotto, Francesco; Saira, Olli-Pentti

\section{Radio-frequency single-electron refrigerator}

Published in:

Physical Review Letters

DOI:

10.1103/PhysRevLett.98.037201

Published: 16/01/2007

Document Version

Publisher's PDF, also known as Version of record

Please cite the original version:

Pekola, J., Giazotto, F., \& Saira, O-P. (2007). Radio-frequency single-electron refrigerator. Physical Review Letters, 98(3), 1-4. [037201]. https://doi.org/10.1103/PhysRevLett.98.037201

This material is protected by copyright and other intellectual property rights, and duplication or sale of all or part of any of the repository collections is not permitted, except that material may be duplicated by you for your research use or educational purposes in electronic or print form. You must obtain permission for any other use. Electronic or print copies may not be offered, whether for sale or otherwise to anyone who is not an authorised user. 


\title{
gु
}

\section{Radio-Frequency Single-Electron Refrigerator}

\author{
Jukka P. Pekola, ${ }^{1}$ Francesco Giazotto, ${ }^{2}$ and Olli-Pentti Saira ${ }^{1}$ \\ ${ }^{1}$ Low Temperature Laboratory, Helsinki University of Technology, P.O. Box 3500, 02015 TKK, Finland \\ ${ }^{2}$ NEST CNR-INFM \& Scuola Normale Superiore, I-56126 Pisa, Italy
}

(Received 3 August 2006; published 16 January 2007)

\begin{abstract}
We propose a cyclic refrigeration principle based on mesoscopic electron transport. Synchronous sequential tunneling of electrons in a Coulomb-blockaded device, a normal metal-superconductor singleelectron box, results in a cooling power of $\sim k_{B} T \times f$ at temperature $T$ over a wide range of cycle frequencies $f$. Electrostatic work, done by the gate voltage source, removes heat from the Coulomb island with an efficiency of $\sim k_{B} T / \Delta$, where $\Delta$ is the superconducting gap parameter. The performance is not affected significantly by nonidealities, for instance by offset charges. We propose ways of characterizing the system and of its practical implementation.
\end{abstract}

DOI: 10.1103/PhysRevLett.98.037201

PACS numbers: 85.80.Fi, 73.23.Hk, 85.25.-j

Cyclic conversion of heat into mechanical work, or conversely, work into extracted heat, demonstrates basic concepts of thermodynamics. The latter forms the basis of refrigeration (see, e.g., [1]). Whereas cryogenic refrigeration dates back to the 19 th century [1,2], electronic microrefrigeration has existed only for a little longer than a decade [3]. Successful solid-state refrigeration schemes operating at cryogenic temperatures, typically in the subKelvin regime, rely most commonly on normal metalinsulator-superconductor (NIS) tunnel junctions [3,4]. They have allowed substantial electron $[5,6]$ as well as phonon $[7,8]$ temperature reduction. Quasiparticle refrigeration occurs thanks to the superconducting energy gap which allows only the most energetic electrons to escape in a tunneling process, thus effectively cooling the $N$ region. Until now no electronic cyclic refrigerators have, to the best of our knowledge, been demonstrated. Yet there exists a proposal to use a mesoscopic semiconductor ratchet as a Brownian heat engine for electrons [9], and very recently a non-adiabatic electron heat pump in an asymmetric resonant structure was described [10]. In this Letter we show that a simple mesoscopic hybrid nanostructure in the Coulomb blockade regime exhibits significant cooling power when work is done on it by a radio-frequency gate, even in the absence of dc bias. The presented concept bears resemblance to that of a single-electron pump, where an electron is transported synchronously at an operation frequency $f$ through a device producing average electric current of magnitude ef [11-13]. In the present system, heat at temperature $T$ is transported similarly due to cyclic gate operation in the Coulomb blockade regime with average "current" of order $k_{B} T f$.

As a basic but representative example we study a singleelectron box (SEB) with a normal metal island and a superconducting lead [see Fig. 1(a)], a system whose electrical properties are determined by single-electron charging effects [14]. In practice it can be easily realized with present-day fabrication technologies. In SEB, a tunnel junction of capacitance $C$ and resistance $R_{T}$ connects the island to the lead. A source providing voltage $V_{g}$ is furthermore capacitively coupled to the island through a capacitance $C_{g}$. The charging energy of the device can be written as $E_{\text {ch }}\left(n, n_{g}\right)=E_{C}\left(n+n_{g}\right)^{2}$. Here, $E_{C}=$ $e^{2} /\left(2 C_{\Sigma}\right)$ is the unit of charging energy, $n$ is the number of extra electrons on the island, and $n_{g}=C_{g} V_{g} / e$ is the amount of charge in units of $e$ induced by the gate on the island. The total capacitance of the island is $C_{\Sigma}=C+$ $C_{g}+C_{0}$, where $C_{0}$ is the self-capacitance of the $N$ region.

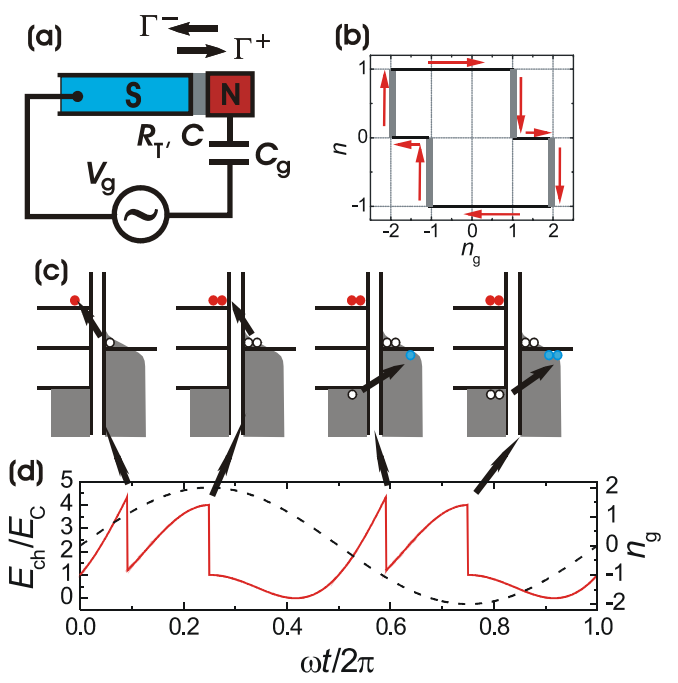

FIG. 1 (color online). Single-electron refrigerator (SER). (a) Single-electron box with a normal metal $(N)$ island and a superconducting $(S)$ lead. (b) The trajectory on the $\left(n, n_{g}\right)$ plane for the cycle discussed here. (c) Sketch of energy band diagrams of the SER showing the tunneling processes in this cycle. In practice, the nonequilibrium excitations relax via inelastic collisions between tunneling events as discussed in the text. (d) The charging energy of the system (solid line, left scale), where discontinuities are observed as electrons tunnel. This change of energy is provided by the voltage source to the tunneling electrons to overcome the superconducting gap. The gate cycle is shown by the dashed line (right scale). 
First we study charge and energy transport in the device, which allows us to obtain the main result of this Letter, i.e., heat extraction in a cyclic operation of the gate voltage. In the following this system will be referred to as a singleelectron refrigerator (SER). We identify two stochastic events, tunneling into the island from the lead, which we associate with a "+" index, and tunneling out from the island to the lead ("-" index). The respective changes in charging energy read

$$
\epsilon^{ \pm} \equiv \delta E_{\mathrm{ch}}^{ \pm} / \Delta= \pm 2 \epsilon_{C}\left(n+n_{g} \pm 1 / 2\right) .
$$

Here, $\Delta$ is the energy gap parameter of the superconductor, and $\epsilon_{C} \equiv E_{C} / \Delta$.

Within the theory of sequential single-electron tunneling [15], assuming that the tunneling electron does not exchange energy with the environment [14], the two rates of electron transport can be written as

$$
\begin{aligned}
& \Gamma^{+}\left(\epsilon^{+}\right)=\frac{\Delta}{e^{2} R_{T}} \int d \epsilon \tilde{n}_{S}(\epsilon) \tilde{f}_{S}(\epsilon)\left[1-\tilde{f}_{N}\left(\epsilon-\epsilon^{+}\right)\right] \\
& \Gamma^{-}\left(\epsilon^{-}\right)=\frac{\Delta}{e^{2} R_{T}} \int d \epsilon \tilde{n}_{S}(\epsilon) \tilde{f}_{N}\left(\epsilon+\epsilon^{-}\right)\left[1-\tilde{f}_{S}(\epsilon)\right] .
\end{aligned}
$$

Here, the normalized BCS density of states at energy $E \equiv$ $\epsilon \Delta$ reads $\tilde{n}_{S}(\epsilon)=\epsilon / \sqrt{\epsilon^{2}-1}$. To take into account nonidealities in realistic superconductors [16], we use $\tilde{n}_{S}(\epsilon)=$ $\left|\operatorname{Re}\left[(\epsilon+i \gamma) / \sqrt{(\epsilon+i \gamma)^{2}-1}\right]\right|$ instead, where the typical value of the smearing parameter $\gamma$ is in the range $1 \times$ $10^{-5} \ldots 1 \times 10^{-3}$ for aluminum as a thin-film superconductor [5]. Furthermore, $\tilde{f}_{S(N)}(\epsilon)$ is the energy distribution function in $S(N)$. With the assumption of quasiequilibrium, as will be explained later in the text, it is given by FermiDirac distribution at temperature $T_{S}(T)$. The corresponding heat fluxes associated with the two tunneling processes are given by

$$
\begin{aligned}
& \dot{Q}^{+}\left(\epsilon^{+}\right)=\frac{\Delta^{2}}{e^{2} R_{T}} \int d \epsilon\left(\epsilon-\epsilon^{+}\right) \tilde{n}_{S}(\epsilon) \tilde{f}_{S}(\epsilon)\left[1-\tilde{f}_{N}\left(\epsilon-\epsilon^{+}\right)\right] \\
& \dot{Q}^{-}\left(\epsilon^{-}\right)=\frac{\Delta^{2}}{e^{2} R_{T}} \int d \epsilon\left(\epsilon+\epsilon^{-}\right) \tilde{n}_{S}(\epsilon) \tilde{f}_{N}\left(\epsilon+\epsilon^{-}\right)\left[1-\tilde{f}_{S}(\epsilon)\right],
\end{aligned}
$$

where the first one is the energy deposition rate by incoming electrons, and the second one is the energy extraction rate by leaving electrons.

We start by an approximate calculation which yields illustrative yet nearly quantitative results. At low temperatures $T, T_{S} \ll \Delta / k_{B}$, and in the domain $1+\epsilon^{ \pm}>0$, Eqs. (2) yield

$$
\Gamma^{ \pm}\left(\epsilon^{ \pm}\right) \simeq \frac{1}{e^{2} R_{T}} \sqrt{\frac{\pi}{2} \Delta k_{B} T} e^{-\left(\Delta / k_{B} T\right)\left(1+\epsilon^{ \pm}\right)} .
$$

With the same approximations, we get from Eqs. (3)

$$
\left\langle Q^{ \pm}\left(\epsilon^{ \pm}\right)\right\rangle \simeq \mp\left[\frac{k_{B} T}{2}+\Delta\left(1+\epsilon^{ \pm}\right)\right]
$$

for the average energy per event of a tunneling electron with respect to the Fermi energy $\left\langle Q^{ \pm}\left(\epsilon^{ \pm}\right)\right\rangle \equiv$ $\dot{Q}^{ \pm}\left(\epsilon^{ \pm}\right) / \Gamma^{ \pm}\left(\epsilon^{ \pm}\right)$. We expect the exponent in Eq. (4) to assume values in the range $1 \ldots 10$ at that point in the cycle where tunneling is most likely to occur. This is because the prefactor in front of the exponential term, the "attempt frequency," is typically a few orders of magnitude higher than the operation frequency of the device [17]. The tunneling electron is then expected to have energy $Q^{ \pm} \sim$ $\mp k_{B} T$. This states that the "+" process brings a negative amount of energy into the island, whereas the "-" process removes a positive energy from the island; i.e., all the events tend to extract hot excitations from the $N$ island, which results in refrigeration of it. The expectation value of energy carried out from the island in a full cycle is then $Q=n_{t}\left(Q^{-}-Q^{+}\right)$, where $n_{t}$ is the number of tunneling events in both directions within a full cycle. We then have $Q=r k_{B} T$, where $r$ is of the order of $n_{t}$. By repeating cycles at frequency $f$ one obtains an average cooling power $\dot{Q}=r k_{B} T f$.

To go beyond these approximate results, we employ either deterministic, master equation-based computations, or stochastic simulations of charge and energy dynamics. In the first method, we follow the probabilities $p_{n}(t)$ of observing charge number $n$ on the island at time instant $t$. Equations (2) yield for each $n$ state the time-dependent transition rates $\Gamma_{n}^{ \pm}$corresponding to the "+" and "-" processes. The master equation then reads

$$
\dot{p}_{n}=-\left(\Gamma_{n}^{+}+\Gamma_{n}^{-}\right) p_{n}+\Gamma_{n-1}^{+} p_{n-1}+\Gamma_{n+1}^{-} p_{n+1} .
$$

Furthermore, we write the expectation value of instantaneous heat current with the help of Eqs. (3) as $P=$ $\sum_{n} p_{n}\left(\dot{Q}_{n}^{-}-\dot{Q}_{n}^{+}\right)$. With a reasonable initial condition, e.g., $p_{n}(0)=\delta_{n 0}, p_{n}(t)$ converges to an approximate periodic equilibrium during the first few cycles. The total cooling power $\dot{Q}$ is then obtained by averaging $P$ over one cycle.

In the stochastic method, we employ a Monte Carlo simulation of the tunnel events to generate a sample trajectory in $n$ space. We evaluate the integrands in Eqs. (2) over a small energy interval $\delta \epsilon$. Multiplied by a small time step $\delta t$, these yield the probability of observing a "+" event adding energy $\epsilon-\epsilon^{+}$or a " - " event removing energy $\epsilon+\epsilon^{-}$during $\delta t$. The total cooling power can be obtained by averaging over a trajectory long enough to suppress the stochastic variation to a negligible level.

As an example, we consider a cycle where the gate voltage varies periodically such that $n_{g}=2 \sin (\omega t)$, see Fig. 1(d). As presented in Fig. 1(b), $n$ then tends to follow the sequence $+1 \rightarrow 0 \rightarrow-1 \rightarrow 0 \rightarrow+1$. The thick vertical lines indicate the stochastic uncertainty of the tunnel events along the gate excursion. In Figs. 1(c) and 1(d) we show, respectively, the tunnel events on an energy diagram 
during the example cycle demonstrating the cooling feature of the device, and the corresponding charging energy along the cycle. Figure 2 shows numerical results over 15 cycles, of charges on the island and heat extracted from it. In the stochastic simulation the ideal cycle of $n:+1 \rightarrow 0 \rightarrow$ $-1 \rightarrow 0 \rightarrow+1$ is repeated almost regularly over these cycles, although the system visits once the $n=-2$ state and the exact time instants of tunneling jitter around their mean values. The deterministic calculation yields also the expectation value of charge on the island at each value of gate (inset). The efficiency of the refrigerator, defined as the ratio of heat extracted and work done by the source, is $\eta \simeq 4.0 k_{B} T / \Delta$ in this particular case. This exceeds by more than a factor of 3 the corresponding figure of a static NIS refrigerator, $\eta \simeq 1.2 k_{B} T / \Delta$ [3]. This difference stems from yet another favorable energy filtering in SER: due to Coulomb blockade, only one electron tunnels in each phase of the cycle, and it extracts maximal amount of heat from $N$.

Further insight into the performance of SER can be gained by inspecting the frequency dependence of $\dot{Q}$, shown in Fig. 3(a) for different values of $R_{T} . \dot{Q}$ is a nonmonotonic function of $f$, decreasing both at low and high values of $f$. Increasing the junction transparency leads to an enhancement of the maximum heat current and to widening of the window of operational frequencies. This can be easily understood in terms of reduced discharging time through the junction. For $R_{T}=30 \mathrm{k} \Omega(5 \mathrm{k} \Omega), \dot{Q}$ is maximized around $f \simeq 150 \mathrm{MHz}(1 \mathrm{GHz})$, where it obtains values of $\sim 1 \mathrm{fW}(\sim 6 \mathrm{fW})$ for Al. As will be shown below, such values will lead to substantial electronic temperature reduction in the $N$ island.

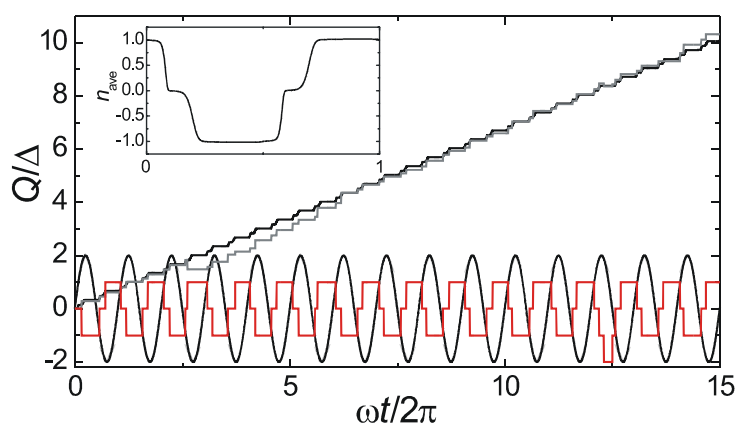

FIG. 2 (color online). Heat extracted from the $N$ island in a SER during the cyclic operation of the gate. These calculations were performed with the following parameter values: $k_{B} T / \Delta=$ $0.05, \epsilon_{C} \equiv E_{C} / \Delta=0.3, \quad R_{T}=30 \mathrm{k} \Omega, \quad \gamma=1 \times 10^{-4}, f \equiv$ $\omega /(2 \pi)=10 \mathrm{MHz}$, and $\Delta=200 \mu \mathrm{eV}$, which corresponds to aluminum as the superconductor. The gray line shows the stochastic results, and the rising black line is the solution of the master equation. The sinusoidal line indicates the instantaneous gate position $n_{g}$, and the piecewise constant red line shows the number of extra electrons on the island in a typical trajectory. The inset displays the average number of charges, $n_{\mathrm{ave}}$, at each instant within the cycle obtained from the master equation.
Whether a single-electron device is usable in practice depends on its immunity to fluctuating background charges $\left(Q_{0}\right)$. Their influence can be studied numerically by adding an offset $n_{0} \equiv Q_{0} / e$ to $n_{g}$. The results are displayed in Fig. 3(b), which indicates heat extracted per cycle vs $n_{0}$ for three values of gate amplitude. Clearly $n_{0}$ has some effect on the cooling power of SER, but these small variations can be compensated for by adjusting either the amplitude or the dc bias of the gate, if necessary. Furthermore, in practice it may be advantageous to suppress the influence of $n_{0}$ by employing larger gate amplitudes at lower $f$. This is because cooling power is approximately proportional to the product of the two, but the relative uncertainty in the number of tunneling electrons in a cycle with larger gate amplitude is smaller.

To test the performance of the SER in a realistic context, we need to take into account the mechanisms that drive power into the $N$ island. We expect the system to be in the quasiequilibrium limit [3], where strong inelastic electronelectron interaction forces the quasiparticle distribution toward a thermal one at a temperature $T$ which can differ from that of the lattice $\left(T_{\text {bath }}\right)$. The inelastic electronelectron relaxation rate is of the order of $\tau_{e^{-e}}^{-1} \sim$ $10^{9} \ldots 10^{10} \mathrm{~s}^{-1}$ in ultrapure metallic samples and faster otherwise [18]. In static NIS refrigerators it has turned out to be very hard to drive the distribution function out from quasiequilibrium [4-6], which happens if the injection rate of electrons exceeds $\tau_{e^{-e}}^{-1}$ significantly. Compared to static coolers, we envision to employ similar $N$ islands, and the typical electron injection rates are at least an order of magnitude lower here. Thus we indeed believe that quasiequilibrium is a good assumption for SER, but if not, a similar analysis as in [5] can be employed to assess the performance in nonequilibrium. The main heat load on electrons comes from phonon scattering with energy exchange according to $\dot{Q}_{e \text {-bath }}\left(T, T_{\text {bath }}\right)=\Sigma \mathcal{V}\left(T^{5}-T_{\text {bath }}^{5}\right)$ [19], where $\Sigma$ is the electron-phonon coupling constant

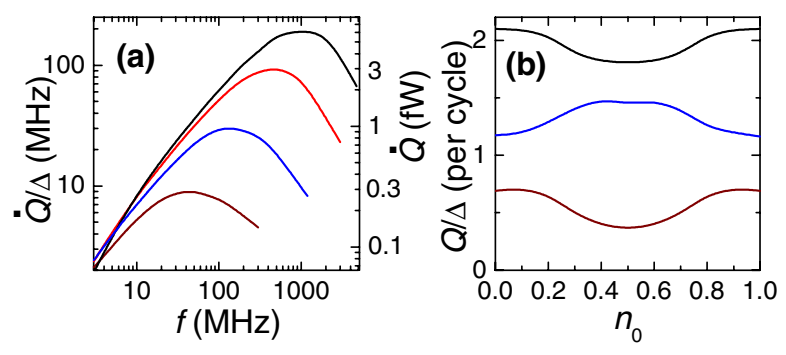

FIG. 3 (color online). (a) Heat flux extracted from the $N$ island of a SER as a function of frequency for some values of $R_{T}$. From bottom to top: $R_{T}=100,30,10$, and $5 \mathrm{k} \Omega$. The computations were performed assuming $n_{g}(t)=2 \sin (\omega t)$. (b) Normalized energy extracted per cycle versus background charge $n_{0}$. In the calculations we used $f=10 \mathrm{MHz}$, and $R_{T}=30 \mathrm{k} \Omega$. The gate amplitudes for the three curves from bottom to top are 2, 3.5, and 5. In both (a) and (b) we assumed $\Delta=200 \mu \mathrm{eV}, k_{B} T / \Delta=$ $0.05, E_{C} / \Delta=0.3$, and $\gamma=1 \times 10^{-4}$. 


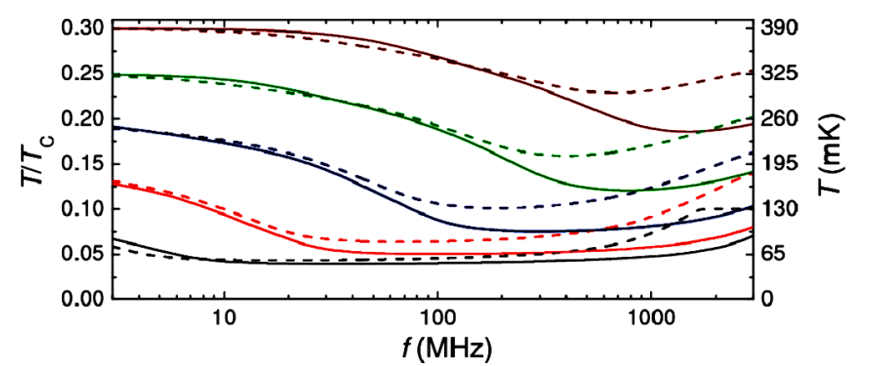

FIG. 4 (color online). Temperature of the $N$ island vs gate frequency at a few bath temperatures. We assume that the volume of the island is $V=1 \times 10^{-21} \mathrm{~m}^{3}, \quad \Sigma=1 \times$ $10^{9} \mathrm{WK}^{-5} \mathrm{~m}^{-3}, \quad E_{C} / \Delta=0.3, \quad$ and $\gamma=1 \times 10^{-4} . \quad T_{C}=$ $\Delta /\left(1.764 k_{B}\right)$ is the superconducting critical temperature. The results are shown for $R_{T}=10 \mathrm{k} \Omega$ (solid lines) and $R_{T}=30 \mathrm{k} \Omega$ (dashed lines), and for bath temperatures (from bottom to top) $131 \mathrm{mK}\left(T_{\text {bath }} / T_{C}=0.1\right.$, black $), 197 \mathrm{mK}\left(T_{\text {bath }} / T_{C}=0.15\right.$, red), $263 \mathrm{mK}\left(T_{\text {bath }} / T_{C}=0.2\right.$, blue $), 328 \mathrm{mK}\left(T_{\text {bath }} / T_{C}=\right.$ 0.25 , green $)$, and $394 \mathrm{mK}\left(T_{\text {bath }} / T_{C}=0.3\right.$, brown).

and $\mathcal{V}$ is the island volume. Heat input by other relaxation mechanisms, in particular, radiative electron-photon heat load, can be ignored in this case because of poor matching $[20,21]$.

To cool the island, frequency $f$ has to be high enough to ensure sufficient cooling power. Just to get an idea one should thus compare $f$ to the inelastic electron-phonon relaxation rate $\tau_{e \text {-ph }}^{-1}$. The latter one has been measured to be $\tau_{e-\mathrm{ph}}^{-1}<10^{5} \mathrm{~Hz}$ at $T \lesssim 200 \mathrm{mK}$ for a similar copper island [22]. In Fig. 4(a) we plot the steady state temperature of an $N$ island of a typical size, obtained as a solution of the balance equation $\dot{Q}(T)+\dot{Q}_{e \text {-bath }}\left(T, T_{\text {bath }}\right)=0$, where $\dot{Q}(T)$ is the cooling power of SER at temperature $T$. We here assume $T_{S}$ to be equal to $T_{\text {bath }}$, but this assumption is not important as long as $T_{S} \lesssim 0.3 T_{C}$ as in a static cooler [3]. The results demonstrate significant temperature reduction over a broad range of frequencies. In practice $T$ can be probed, e.g., by an additional NIS junction, measuring the tiny quasiparticle current averaged over radiofrequency gate modulation. A thermometer based on supercurrent in a Coulomb-blockaded SINIS (two NIS junctions) is another possibility [23]. The performance of a SER can be tested also by detecting the drop of lattice temperature in a membrane configuration, as has been done with static refrigerators in $[7,8]$.

The concept presented here can readily be generalized to more complex structures with further enhanced performance. For example, a three terminal device, in form of a SINIS single-electron transistor, can be controlled additionally by applying a drain-to-source voltage across. Coulomb blockade suppresses the cooling power of a statically biased SINIS refrigerator [24], but adding radio-frequency gating can enhance the cooling power even above that of the SER presented here. Finally, we analyzed here quantitatively only sinusoidal cycles of $n_{g}$ in the basic SER. Alternative architectures and cycles may lead to better control between $\dot{Q}$ and $k_{B} T f$, and the device might then yield a "quantized" and synchronized heat current in analogy to electrical current in metrological devices.

We thank Mikko Paalanen and Matthias Meschke for helpful discussions and Academy of Finland (TULE) and the EC-funded ULTI Project, Transnational Access in Programme FP6 (Contract No. RITA-CT-2003-505313) for financial support.

[1] L.E. Reichl, A Modern Course in Statistical Physics (Wiley, New York, 1998), 2nd ed.

[2] F. Pobell, Matter and Methods at Low Temperatures (Springer-Verlag, Berlin-Heidelberg, 1996), 2nd ed.

[3] F. Giazotto et al., Rev. Mod. Phys. 78, 217 (2006).

[4] M. Nahum, T. M. Eiles, and J. M. Martinis, Appl. Phys. Lett. 65, 3123 (1994).

[5] J. P. Pekola et al., Phys. Rev. Lett. 92, 056804 (2004).

[6] M. M. Leivo, J. P. Pekola, and D. V. Averin, Appl. Phys. Lett. 68, 1996 (1996).

[7] A. Luukanen et al., J. Low Temp. Phys. 120, 281 (2000).

[8] A. M. Clark et al., Appl. Phys. Lett. 86, 173508 (2005).

[9] T. E. Humphrey et al., Phys. Rev. Lett. 89, 116801 (2002).

[10] M. Rey et al., cond-mat/0610155.

[11] H. Pothier et al., Europhys. Lett. 17, 249 (1992).

[12] M. W. Keller et al., Appl. Phys. Lett. 69, 1804 (1996).

[13] M. W. Keller et al., Science 285, 1706 (1999).

[14] For a collection of reviews, see Single Charge TunnelingCoulomb Blockade Phenomena in Nanostructures, edited by H. Grabert and M.H. Devoret (Plenum, New York, 1992).

[15] D. V. Averin and K. K. Likharev, J. Low Temp. Phys. 62, 345 (1986).

[16] R. C. Dynes et al., Phys. Rev. Lett. 53, 2437 (1984).

[17] We may assume $k_{B} T / \Delta \sim 0.1$ for temperature to be well below the superconducting transition temperature $T_{C}(\simeq 1 \mathrm{~K}$ for a usual superconductor such as $\mathrm{Al})$, and $R_{T} \simeq h / e^{2} \simeq 26 \mathrm{k} \Omega$ for junction to be in the Coulomb blockade regime. Then $\frac{1}{e^{2} R_{T}} \sqrt{\frac{\pi}{2} \Delta k_{B} T} \sim 10 \mathrm{GHz}$, which is well above the operation frequencies that we envision here, say $1 \mathrm{MHz} . .1 \mathrm{GHz}$. Thereby our argument is quite generally valid.

[18] F. Pierre et al., Phys. Rev. B 68, 085413 (2003).

[19] M. L. Roukes et al., Phys. Rev. Lett. 55, 422 (1985).

[20] D. R. Schmidt, R. J. Schoelkopf, and A. N. Cleland, Phys. Rev. Lett. 93, 045901 (2004).

[21] M. Meschke, W. Guichard, and J.P. Pekola, Nature (London) 444, 187 (2006).

[22] L. J. Taskinen et al., Phys. Status Solidi C 1, 2856 (2004).

[23] P. M. Ostrovsky and M. V. Feigel'man, Pis'ma Zh. Eksp. Teor. Fiz. 82, 863 (2005) [JETP Lett. 82, 763 (2005)].

[24] J.P. Pekola and F. Giazotto (unpublished). 